University of South Florida

DIGITAL COMMONS

Digital Commons @ University of

@ UNIVERSITY OF SOUTH FLORIDA

South Florida

$1-1-2019$

\title{
2019 Accountability Plan Sarasota-Manatee
}

USF

Follow this and additional works at: https://digitalcommons.usf.edu/usf_accountability_reports

\section{Scholar Commons Citation}

USF, "2019 Accountability Plan Sarasota-Manatee" (2019). USF Accountability Reports. 73.

https://digitalcommons.usf.edu/usf_accountability_reports/73

This Article is brought to you for free and open access by the USF Archives at Digital Commons @ University of South Florida. It has been accepted for inclusion in USF Accountability Reports by an authorized administrator of Digital Commons @ University of South Florida. For more information, please contact digitalcommons@usf.edu. 


\section{9 \\ Accountability Plan}

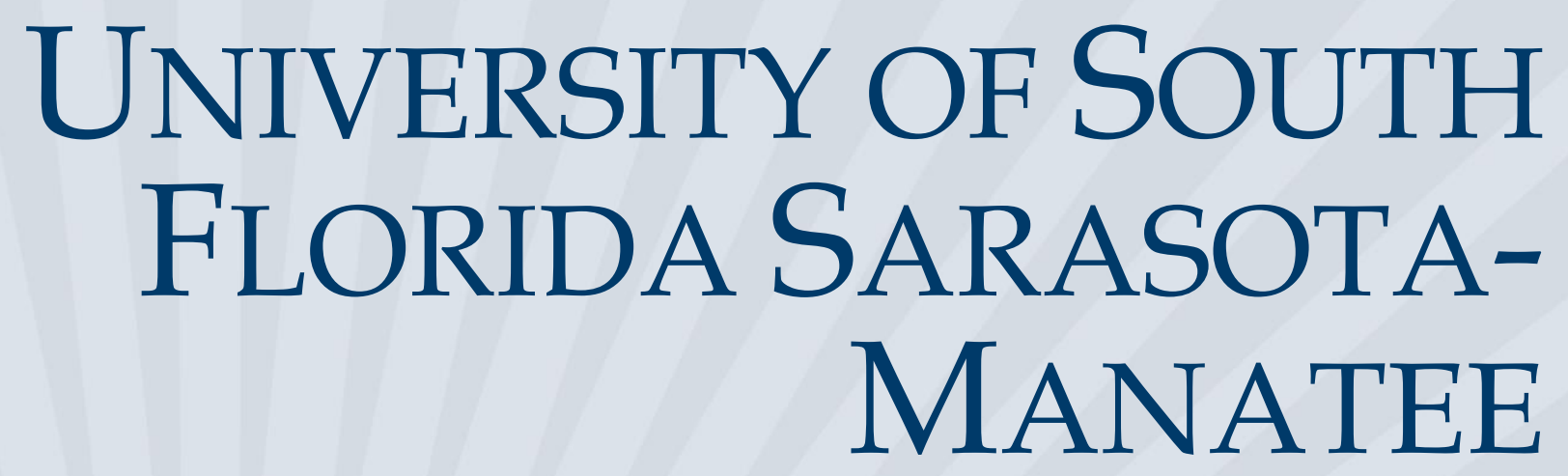

FINAL FOR BOG SUBMISSION

STATE UNIVERSITY SYSTEM of FLORIDA Board of Governors 


\section{INTRODUCTION}

This is a new report that combines the previous Annual Accountability Report and University Work Plans into a single document more closely aligned with the Board of Governors' 2025 System Strategic Plan.

This revised document will enhance the System's commitment to accountability and strategic planning by enabling comparisons between past goals and actual data to better assess performance, helping to foster greater coordination between institutional administrators, University Boards of Trustees, and the Board of Governors.

Once an Accountability Plan is approved by each institution's respective Boards of Trustees, the Board of Governors will review and consider the plan for approval, excluding those sections of the Plan that require additional regulatory or procedural approval pursuant to law or Board regulations. 


\section{TABLE OF CONTENTS}

1. PERFORMANCE BASED FUNDING METRICS, p. 3-4

2. KEY PERFORMANCE INDICATORS

a. Teaching \& Learning, p. 5-6

b. Institution Specific Goals, p. 7

3. ENROLLMENT PLANNING, p. 8-9 


\section{PERFORMANCE BASED FUNDING METRICS}

\begin{tabular}{|c|c|c|c|c|c|c|c|c|c|}
\hline & $2012-13$ & 2013-14 & 2014-15 & $2015-16$ & 2016-17 & 2017-18 & 2018-19 & $2019-20$ & 2020-21 \\
\hline ACTUAL & 65.5 & 62.8 & 69.6 & 71.7 & 70.6 & . & . & . & . \\
\hline APPROVED GOALS & . & . & 63.5 & 72.8 & 72.8 & 73.8 & 74.8 & 75.8 & . \\
\hline PROPOSED GOALS & . & . & . & . & . & 72.8 & 73.8 & 74.8 & 75.8 \\
\hline
\end{tabular}

2. Median Wages of Bachelor's Graduates Employed Full-time

\begin{tabular}{cccccccccc} 
& $2012-13$ & $2013-14$ & $2014-15$ & $2015-16$ & $2016-17$ & $2017-18$ & $2018-19$ & $2019-20$ & $2020-21$ \\
\hline ACTUAL & 36,000 & 36,200 & 39,000 & 37,000 & 38,200 &. &. &. &. \\
APPROVED GOALS &. &. &. & 40,700 & 38,200 & 38,900 & 39,500 & 40,700 &. \\
PROPOSED GOALS &. &. &. &. &. & 38,900 & 39,900 & 40,900 & 41,000
\end{tabular}

3. Average Cost to the Student [Net Tuition \& Fees per 120 Credit Hours for Resident Undergraduates]

\begin{tabular}{|c|c|c|c|c|c|c|c|c|c|}
\hline & 2013-14 & 2014-15 & 2015-16 & 2016-17 & $2017-18$ & 2018-19 & 2019-20 & $2020-21$ & 2021-22 \\
\hline ACTUAL & & & & & & & & & \\
\hline APPROVED GOALS & \multirow{2}{*}{\multicolumn{9}{|c|}{ Data reported at the USF System level only. }} \\
\hline PROPOSED GOALS & & & & & & & & & \\
\hline \multicolumn{10}{|c|}{ 4. FTIC Four-Year Graduation Rate } \\
\hline & 2010-14 & 2011-15 & 2012-16 & 2013-17 & 2014-18 & 2015-19 & 2016-20 & $2017-21$ & 2018-22 \\
\hline $\begin{array}{l}\text { ACTUAL Graduated } \\
\text { Within USF System }\end{array}$ & & & & 45.8 & 40.4 & & & & \\
\hline $\begin{array}{l}\text { ACTUAL Graduated } \\
\text { Same Campus }\end{array}$ & & . & . & 34.9 & 31.1 & . & . & 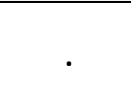 & \\
\hline APPROVED GOALS & . & . & . & . & 36.0 & 40.0 & 45.0 & 50.0 & . \\
\hline PROPOSED GOALS & . & . & . & . & . & 42.0 & 50.0 & 55.0 & 60.0 \\
\hline
\end{tabular}

5. Academic Progress Rate [Second Year Retention Rate with At Least a 2.0 GPA]

\begin{tabular}{|c|c|c|c|c|c|c|c|c|c|}
\hline & 2013-14 & 2014-15 & 2015-16 & 2016-17 & 2017-18 & 2018-19 & $2019-20$ & 2020-21 & 2021-22 \\
\hline $\begin{array}{l}\text { ACTUAL Graduated } \\
\text { Within USF System }\end{array}$ & 77.1 & 86.7 & 78.2 & 83.3 & 84.7 & . & . & . & . \\
\hline $\begin{array}{l}\text { ACTUAL Graduated } \\
\text { Same Campus }\end{array}$ & 69.9 & 78.7 & 74.7 & $81.3^{*}$ & 84.0 & & & & \\
\hline APPROVED GOALS & . & . & 80.2 & 82.0 & 84.4 & 86.4 & 88.4 & 90.0 & . \\
\hline PROPOSED GOALS & . & . & . & . & . & 86.4 & 89.0 & 90.0 & 91.0 \\
\hline
\end{tabular}

Note*: Previous year data updated to reflect the change in methodology made by Board ODA staff to improve accuracy.

Note: Metrics are defined in appendix. For more information about the PBF model visit: http://www.flbog.edu/about/budget/performance funding.php. 
6. Percentage of Bachelor's Degrees Awarded within Programs of Strategic Emphasis

\begin{tabular}{|c|c|c|c|c|c|c|c|c|c|}
\hline & 2013-14 & 2014-15 & 2015-16 & 2016-17 & 2017-18 & 2018-19 & 2019-20 & 2020-21 & 2021-22 \\
\hline ACTUAL & 33.9 & 41.1 & 37.0 & 39.1 & 43.7 & & & . & . \\
\hline APPROVED GOALS & & • & 42.0 & 42.0 & 44.1 & 47.0 & 49.0 & 50.0 & . \\
\hline PROPOSED GOALS & . & & & . & . & 44.3 & 49.0 & 50.0 & 50.0 \\
\hline \multicolumn{10}{|c|}{ 7. University Access Rate [Percent of Undergraduates with a Pell grant] } \\
\hline & $\begin{array}{l}\text { FALL } \\
2013 \\
\end{array}$ & $\begin{array}{l}\text { FALL } \\
2014 \\
\end{array}$ & $\begin{array}{l}\text { FALL } \\
2015 \\
\end{array}$ & $\begin{array}{l}\text { FALL } \\
2016 \\
\end{array}$ & $\begin{array}{l}\text { FALL } \\
2017 \\
\end{array}$ & $\begin{array}{l}\text { FALL } \\
2018 \\
\end{array}$ & $\begin{array}{l}\text { FALL } \\
2019 \\
\end{array}$ & $\begin{array}{l}\text { FALL } \\
2020 \\
\end{array}$ & $\begin{array}{l}\text { FALL } \\
2021 \\
\end{array}$ \\
\hline ACTUAL & 40.7 & 45.2 & 44.4 & 41.1 & 44.6 & & & . & . \\
\hline APPROVED GOALS & & . & 41.0 & 44.4 & 44.4 & 45.0 & 45.0 & 45.0 & . \\
\hline PROPOSED GOALS & . & . & & . & . & 45.0 & 45.0 & 45.0 & 45.0 \\
\hline
\end{tabular}

8. Percentage of Graduate Degrees Awarded within Programs of Strategic Emphasis

\begin{tabular}{cccccccccc} 
& $2013-14$ & $2014-15$ & $2015-16$ & $2016-17$ & $2017-18$ & $2018-19$ & $\mathbf{2 0 1 9 - 2 0}$ & $\mathbf{2 0 2 0 - 2 1}$ & $\mathbf{2 0 2 1 - 2 2}$ \\
\hline ACTUAL & 27.3 & 12.0 & 24.5 & 11.7 & 21.7 &. &. &. &. \\
APPROVED GOALS &. &. & 19.0 & 12.0 & 20.0 & 25.0 & 30.0 & 35.0 &. \\
PROPOSED GOALS &. &. &. &. &. & 16.7 & 19.0 & 21.0 & 23.0
\end{tabular}

\section{BOG Choice: Percent of Baccalaureate Degrees Awarded Without Excess Hours}

\begin{tabular}{cccccccccc} 
& $2013-14$ & $2014-15$ & $2015-16$ & $2016-17$ & $2017-18$ & $2018-19$ & $2019-20$ & $2020-21$ & $2021-22$ \\
\hline ACTUAL & 75.4 & 71.7 & 75.7 & 80.3 & 78.5 &. &. &. &. \\
APPROVED GOALS &. &. & 72.9 & 78.0 & 81.0 & 82.0 & 83.0 & 84.0 &. \\
PROPOSED GOALS &. &. &. &. &. & 81.0 & 82.0 & 83.0 & 84.0
\end{tabular}

10.1 Current BOT Choice: Number of Post-Doctoral Appointees

\begin{tabular}{cccccccccc} 
& 2013 & 2014 & 2015 & 2016 & 2017 & 2018 & 2019 & 2020 & 2021 \\
\hline ACTUAL &. & 0 & 5 & 5 & $4^{*}$ &. &. &. &. \\
APPROVED GOALS &. &. &. & 5 & 5 & 5 & 5 & 5 &. \\
PROPOSED GOALS &. &. &. &. &. & 5 & 5 & 5 & 5
\end{tabular}

Note*: Revised since reported to NSF

10.2 Future BOT Choice: Six-Year FTIC Graduation Rate [Full \& Part-Time Students] $\begin{array}{lllllllll}2008-14 & 2009-15 & 2010-16 & 2011-17 & 2012-18 & 2013-19 & 2014-20 & 2015-21 & 2016-22\end{array}$

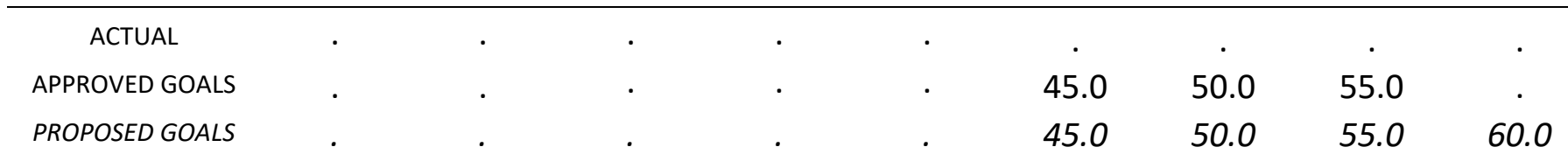

Note: This is a transition year for the BOT Choice metric (\#10), so we are reporting data for both the current and future metrics. Metrics are defined in appendix. For more information about the PBF model visit: http://www.flbog.edu/about/budget/performance funding.php 


\section{KEY PERFORMANCE INDICATORS}

Teaching \& Learning Metrics (from the 2025 System Strategic Plan that are not included in the PBF section) Public University National Ranking [Number of Top50 Rankings based on BOG's official list of publications]

\begin{tabular}{|c|c|c|c|c|c|c|c|c|c|}
\hline & 2015 & 2016 & 2017 & 2018 & 2019 & 2020 & 2021 & 2022 & 2023 \\
\hline ACTUAL & & & & & & & & & \\
\hline APPROVED GOALS & \multicolumn{9}{|c|}{ Data reported at the USF System level only. } \\
\hline \multicolumn{10}{|l|}{ PROPOSED GOALS } \\
\hline \multicolumn{10}{|c|}{ Freshmen in Top 10 of High School Class } \\
\hline & Fall 2014 & Fall 2015 & Fall 2016 & Fall 2017 & Fall 2018 & Fall 2019 & Fall 2020 & Fall 2021 & Fall 2022 \\
\hline ACTUAL & 30 & 26 & 29 & 16 & 18 & . & . & . & \\
\hline APPROVED GOALS & . & . & 26 & 30 & 25 & 30 & 32 & 35 & . \\
\hline PROPOSED GOALS & . & . & . & . & . & 30 & 32 & 35 & 35 \\
\hline
\end{tabular}

Time to Degree for FTICs in 120 hr programs

\begin{tabular}{cccccccccc} 
& $2013-14$ & $2014-15$ & $2015-16$ & $2016-17$ & $2017-18$ & $2018-19$ & $2019-20$ & $2020-21$ & $2021-22$ \\
\hline ACTUAL &. &. &. & 3.4 & 3.5 &. &. &. &. \\
APPROVED GOALS &. &. &. & 4.2 & 4.0 & 4.0 & 4.0 & 4.0 &. \\
PROPOSED GOALS &. &. &. &. &. & 4.0 & 4.0 & 4.0 & 4.0
\end{tabular}

Six-Year FTIC Graduation Rates [full-\& part-time students]

\begin{tabular}{cccccccccc} 
& $2008-14$ & $2009-15$ & $2010-16$ & $2011-17$ & $2012-18$ & $2013-19$ & $2014-20$ & $2015-21$ & $2016-22$ \\
\hline $\begin{array}{c}\text { ACTUAL Graduated } \\
\text { Same Campus }\end{array}$ &. &. &. &. &. &. &. &. &. \\
APPROVED GOALS &. &. &. &. &. & 45.0 & 50.0 & 55.0 &. \\
PROPOSED GOALS &. &. &. &. &. & 45.0 & 50.0 & 55.0 & 60.0
\end{tabular}

Bachelor's Degrees Awarded [First Majors Only]

\begin{tabular}{cccccccccc} 
& $2013-14$ & $2014-15$ & $2015-16$ & $2016-17$ & $2017-18$ & $\mathbf{2 0 1 8 - 1 9}$ & $\mathbf{2 0 1 9 - 2 0}$ & $\mathbf{2 0 2 0 - 2 1}$ & $\mathbf{2 0 2 1 - 2 2}$ \\
\hline ACTUAL & 490 & 474 & 464 & 460 & 488 &. &. &. &. \\
APPROVED GOALS &. &. & 495 & 442 & 485 & 502 & 522 & 553 &. \\
PROPOSED GOALS &. &. &. &. &. & 522 & 532 & 553 & 564
\end{tabular}




\section{KEY PERFORMANCE INDICATORS (CONTINUED)}

\section{Teaching \& Learning Metrics}

Graduate Degrees Awarded [First Majors Only]

\begin{tabular}{cccccccccc} 
& $2013-14$ & $2014-15$ & $2015-16$ & $2016-17$ & $\mathbf{2 0 1 7 - 1 8}$ & $\mathbf{2 0 1 8 - 1 9}$ & $\mathbf{2 0 1 9 - 2 0}$ & $\mathbf{2 0 2 0 - 2 1}$ & $\mathbf{2 0 2 1 - 2 2}$ \\
\hline ACTUAL & 55 & 50 & 53 & 77 & 69 &. &. &. &. \\
APPROVED GOALS &. &. & 52 & 73 & 78 & 81 & 84 & 89 &. \\
PROPOSED GOALS &. &. &. &. &. & 48 & 49 & 52 & 57
\end{tabular}

Percent of Bachelor's Degrees Awarded to African-American \& Hispanic Students

\begin{tabular}{cccccccccc} 
& $2013-14$ & $2014-15$ & $2015-16$ & $2016-17$ & $2017-18$ & $2018-19$ & $2019-20$ & $2020-21$ & $2021-22$ \\
\hline ACTUAL & 15 & 24 & 21 & 17 & 20 &. &. &. &. \\
APPROVED GOALS &. &. & 23 & 21 & 21 & 22 & 23 & 24 &. \\
PROPOSED GOALS &. &. &. &. &. & 22 & 23 & 24 & 25
\end{tabular}

Percent of Adult (Aged 25+) Undergraduates Enrolled

$\begin{array}{lllllllll}\text { Fall } 2014 & \text { Fall } 2015 & \text { Fall } 2016 & \text { Fall } 2017 & \text { Fall } 2018 & \text { Fall } 2019 & \text { Fall } 2020 & \text { Fall } 2021 & \text { Fall } 2022\end{array}$

\begin{tabular}{cccccccccccc}
\hline ACTUAL & 51 & 48 & 44 & 44 & 43 &. &. &. &. \\
APPROVED GOALS &. &. & 50 & 43 & 43 & 42 & 42 & 41 & . \\
PROPOSED GOALS &. &. &. &. &. & 42 & 42 & 41 & 41
\end{tabular}

Percent of Undergraduate FTE in Online Courses

\begin{tabular}{cccccccccc} 
& $\mathbf{2 0 1 3 - 1 4}$ & $\mathbf{2 0 1 4 - 1 5}$ & $\mathbf{2 0 1 5 - 1 6}$ & $\mathbf{2 0 1 6 - 1 7}$ & $\mathbf{2 0 1 7 - 1 8}$ & $\mathbf{2 0 1 8 - 1 9}$ & $\mathbf{2 0 1 9 - 2 0}$ & $\mathbf{2 0 2 0 - 2 1}$ & $\mathbf{2 0 2 1 - 2 2}$ \\
\hline ACTUAL & 50 & 49 & 52 & 60 & 63 &. &. &. &. \\
APPROVED GOALS &. &. & 52 & 53 & 63 & 60 & 55 & 55 &. \\
PROPOSED GOALS &. &. &. &. &. & 63 & 63 & 63 & 63
\end{tabular}

Percent of Bachelor's Degrees in STEM \& Health

\begin{tabular}{cccccccccc} 
& $\mathbf{2 0 1 3 - 1 4}$ & $\mathbf{2 0 1 4 - 1 5}$ & $\mathbf{2 0 1 5 - 1 6}$ & $\mathbf{2 0 1 6 - 1 7}$ & $\mathbf{2 0 1 7 - 1 8}$ & $\mathbf{2 0 1 8 - 1 9}$ & $\mathbf{2 0 1 9 - 2 0}$ & $\mathbf{2 0 2 0 - 2 1}$ & $\mathbf{2 0 2 1 - 2 2}$ \\
\hline ACTUAL & 14 & 19 & 15 & 22 & 21 &. &. &. &. \\
APPROVED GOALS &. &. & 20 & 19 & 23 & 24 & 25 & 26 &. \\
PROPOSED GOALS &. &. &. &. &. & 21 & 23 & 24 & 25
\end{tabular}

Percent of Graduate Degrees in STEM \& Health

\begin{tabular}{lllllllll}
$2013-14$ & $2014-15$ & $2015-16$ & $2016-17$ & $2017-18$ & $2018-19$ & $2019-20$ & $2020-21$ & $2021-22$ \\
\hline
\end{tabular}

ACTUAL

APPROVED GOALS

PROPOSED GOALS 


\section{Institution Specific Goals}

To further distinguish the university's distinctive mission, the university may choose to provide additional metric goals that are based on the university's own strategic plan.

1. Percent of Non-White Faculty \& Staff

\begin{tabular}{ccccc|cccc}
$\begin{array}{c}\text { Fall 2014 } \\
\text { ACTUAL }\end{array}$ & $\begin{array}{c}\text { Fall 2015 } \\
\text { ACTUAL }\end{array}$ & $\begin{array}{c}\text { Fall 2016 } \\
\text { ACTUAL }\end{array}$ & $\begin{array}{c}\text { Fall 2017 } \\
\text { ACTUAL }\end{array}$ & $\begin{array}{c}\text { Fall 2018 } \\
\text { ACTUAL }\end{array}$ & $\begin{array}{c}\text { Fall 2019 } \\
\text { GOAL }\end{array}$ & $\begin{array}{c}\text { Fall 2020 } \\
\text { GOAL }\end{array}$ & $\begin{array}{c}\text { Fall 2021 } \\
\text { GOAL }\end{array}$ & $\begin{array}{c}\text { Fall } 2022 \\
\text { GOAL }\end{array}$ \\
\hline 18 & 19 & 20 & 25 & 17 & 20 & 21 & 22 & 23
\end{tabular}

2. Percent of Hispanic \& African American Students

\begin{tabular}{ccccc|cccc}
$\begin{array}{c}\text { Fall 2014 } \\
\text { ACTUAL }\end{array}$ & $\begin{array}{c}\text { Fall 2015 } \\
\text { ACTUAL }\end{array}$ & $\begin{array}{c}\text { Fall 2016 } \\
\text { ACTUAL }\end{array}$ & $\begin{array}{c}\text { Fall 2017 } \\
\text { ACTUAL }\end{array}$ & $\begin{array}{c}\text { Fall 2018 } \\
\text { ACTUAL }\end{array}$ & $\begin{array}{c}\text { Fall 2019 } \\
\text { GOAL }\end{array}$ & $\begin{array}{c}\text { Fall 2020 } \\
\text { GOAL }\end{array}$ & $\begin{array}{c}\text { Fall 2021 } \\
\text { GOAL }\end{array}$ & $\begin{array}{c}\text { Fall } 2022 \\
\text { GOAL }\end{array}$ \\
\hline 20 & 20 & 19 & 21 & 23 & 24 & 25 & 26 & 27
\end{tabular}




\section{ENROLLMENT PLANNING}

Fall Headcount Enrollment by Student Level (for all degree-seeking students at all campuses)

\begin{tabular}{|c|c|c|c|c|c|c|c|c|c|}
\hline & 2014 & 2015 & 2016 & 2017 & 2018 & 2019 & 2020 & 2021 & 2022 \\
\hline \multicolumn{10}{|l|}{ UNDERGRADUATE } \\
\hline ACTUAL & 1,692 & 1,757 & 1,794 & 1,870 & 1,838 & . & . & . & . \\
\hline APPROVED GOALS & . & . & . & 1,878 & 1,974 & 2,143 & 2,342 & 2,568 & . \\
\hline PROPOSED GOALS & . & . & . & . & . & 1,909 & 2,057 & 2,246 & 2,489 \\
\hline \multicolumn{10}{|l|}{ GRADUATE } \\
\hline ACTUAL & 120 & 174 & 178 & 149 & 142 & . & . & . & . \\
\hline APPROVED GOALS & . & . & . & 186 & 154 & 163 & 172 & 185 & . \\
\hline PROPOSED GOALS & . & . & . & . & . & 150 & 165 & 181 & 199 \\
\hline
\end{tabular}

Fall Headcount Enrollment by Student Type (for all degree-seeking students at all campuses)

\begin{tabular}{|c|c|c|c|c|c|c|c|c|c|}
\hline & $\begin{array}{c}2014 \\
\text { ACTUAL }\end{array}$ & $\begin{array}{c}2015 \\
\text { ACTUAL }\end{array}$ & $\begin{array}{c}2016 \\
\text { ACTUAL }\end{array}$ & $\begin{array}{c}2017 \\
\text { ACTUAL }\end{array}$ & $\begin{array}{c}2018 \\
\text { ACTUAL }\end{array}$ & $\begin{array}{l}2019 \\
\text { PLAN }\end{array}$ & $\begin{array}{l}2020 \\
\text { PLAN }\end{array}$ & $\begin{array}{l}2021 \\
\text { PLAN }\end{array}$ & $\begin{array}{l}2022 \\
\text { PLAN }\end{array}$ \\
\hline \multicolumn{10}{|l|}{ UNDERGRADUATE } \\
\hline FTIC & 196 & 271 & 321 & 368 & 382 & 393 & 433 & 519 & 649 \\
\hline FCS AA Transfers & 789 & 781 & 737 & 759 & 732 & 791 & 885 & 974 & 1071 \\
\hline Other AA Transfers & 155 & 159 & 159 & 158 & 150 & 158 & 173 & 191 & 210 \\
\hline Post-Baccalaureates & 152 & 127 & 137 & 102 & 90 & 93 & 102 & 108 & 115 \\
\hline Other Undergraduates & 400 & 419 & 440 & 483 & 484 & 474 & 464 & 454 & 444 \\
\hline Subtotal & 1,692 & 1,757 & 1,794 & 1,870 & 1,838 & 1,909 & 2,057 & 2,246 & 2,489 \\
\hline \multicolumn{10}{|l|}{ GRADUATE } \\
\hline Master's & 120 & 174 & 178 & 149 & 142 & 150 & 165 & 181 & 199 \\
\hline Research Doctoral & . & . & . & . & . & . & . & . & . \\
\hline Professional Doctoral & . & & . & . & . & . & . & . & . \\
\hline Subtotal & 120 & 174 & 178 & 149 & 142 & 150 & 165 & 181 & 199 \\
\hline TOTAL & 1,812 & 1,931 & 1,972 & 2,019 & 1,980 & 2,059 & 2,222 & 2,427 & 2,688 \\
\hline
\end{tabular}

Notes: This table reports the number of students enrolled at the university by student type categories. The student type for undergraduates is based on the Type of Student at Time of Most Recent Admission. The student type for graduates is based on the degree that is sought and the student CIP code. Does not include 'Unclassified' students who are not formally admitted into a degree program but are enrolled (e.g., dual enrolled high school students).

\section{Percent of Baccalaureate-Seeking Resident Undergraduates Who Earned 15+ Credit Hours} (Fall terms only)

\begin{tabular}{ccccccccccc} 
& 2014 & 2015 & 2016 & 2017 & 2018 & 2019 & 2020 & 2021 & 2022 \\
\hline ACTUAL & 9 & 10 & 10 & 9 & 9 &. &. &. &. \\
APPROVED GOALS &. &. &. &. &. &. &. &. &. & 20 \\
PROPOSED GOALS &. &. &. &. &. & 12 & 14 & 20
\end{tabular}


FINAL FOR BOG SUBMISSION

BOG Deadline: 05/06/2019

\section{ENROLLMENT PLANNING continued}

\begin{tabular}{|c|c|c|c|c|c|c|c|c|c|c|}
\hline & $\begin{array}{l}2013-14 \\
\text { ACTUAL }\end{array}$ & $\begin{array}{l}2014-15 \\
\text { ACTUAL }\end{array}$ & $\begin{array}{l}\text { 2015-16 } \\
\text { ACTUAL }\end{array}$ & $\begin{array}{l}2016-17 \\
\text { ACTUAL }\end{array}$ & $\begin{array}{l}2017-18 \\
\text { ACTUAL }\end{array}$ & $\begin{array}{c}2018-19 \\
\text { PLAN }\end{array}$ & $\begin{array}{c}2019-20 \\
\text { PLAN }\end{array}$ & $\begin{array}{c}2020-21 \\
\text { PLAN }\end{array}$ & $\begin{array}{c}2021-22 \\
\text { PLAN }\end{array}$ & $\begin{array}{c}2022-23 \\
\text { PLAN }\end{array}$ \\
\hline \multicolumn{11}{|c|}{ RESIDENT } \\
\hline LOWER & 279 & 342 & 398 & 465 & 579 & 623 & 663 & 696 & 738 & 782 \\
\hline UPPER & 1,111 & 1,103 & 1,175 & 1,159 & 1,141 & 1,182 & 1,235 & 1,297 & 1,375 & 1,457 \\
\hline GRAD I & 127 & 120 & 147 & 141 & 124 & 116 & 120 & 124 & 128 & 133 \\
\hline GRAD ॥ & 0 & 0 & 0 & 0 & 0 & 1 & 0 & 0 & 0 & 0 \\
\hline TOTAL & 1,517 & 1,566 & 1,720 & 1,765 & 1,844 & 1,921 & 2,018 & 2,117 & 2,241 & 2,372 \\
\hline \multicolumn{11}{|c|}{ NON-RESIDENT } \\
\hline LOWER & 16 & 22 & 33 & 35 & 44 & 57 & 58 & 61 & 64 & 68 \\
\hline UPPER & 40 & 51 & 68 & 63 & 66 & 76 & 79 & 83 & 88 & 93 \\
\hline GRAD I & 7 & 8 & 15 & 13 & 9 & 5 & 5 & 5 & 5 & 6 \\
\hline GRAD ॥ & 0 & 0 & 0 & 0 & 0 & 0 & 0 & 0 & 0 & 0 \\
\hline TOTAL & 63 & 81 & 116 & 111 & 119 & 138 & 141 & 148 & 157 & 167 \\
\hline \multicolumn{11}{|l|}{ TOTAL } \\
\hline LOWER & 296 & 365 & 431 & 500 & 623 & 680 & 721 & 757 & 802 & 850 \\
\hline UPPER & 1,151 & 1,155 & 1,243 & 1,222 & 1,206 & 1,257 & 1,314 & 1,380 & 1,462 & 1,550 \\
\hline GRAD I & 134 & 127 & 162 & 154 & 133 & 121 & 125 & 129 & 134 & 138 \\
\hline GRAD ॥ & 0 & 1 & 1 & 0 & 0 & 1 & 0 & 0 & 0 & 0 \\
\hline TOTAL & 1,581 & 1,647 & 1,836 & 1,876 & 1,962 & 2,059 & 2,159 & 2,265 & 2,398 & 2,539 \\
\hline
\end{tabular}

Note: Full-time Equivalent (FTE) student is a measure of all instructional activity (regardless of fundability) that is based on the number of credit hours that degreeseeking students enroll. FTE is based on the standard national definition, which divides undergraduate credit hours by 30 and graduate credit hours by 24 . Pursuant to section 1013.31, Florida Statutes, Board facilities staff use this data as a key factor in the calculation of facility space needs for university educational plant surveys.

\begin{tabular}{|c|c|c|c|c|c|c|c|c|c|c|}
\hline & $\begin{array}{l}2013-14 \\
\text { ACTUAL }\end{array}$ & $\begin{array}{l}2014-15 \\
\text { ACTUAL }\end{array}$ & $\begin{array}{l}2015-16 \\
\text { ACTUAL }\end{array}$ & $\begin{array}{l}2016-17 \\
\text { ACTUAL }\end{array}$ & $\begin{array}{l}2017-18 \\
\text { ACTUAL }\end{array}$ & $\begin{array}{c}2018-19 \\
\text { PLAN }\end{array}$ & $\begin{array}{c}2019-20 \\
\text { PLAN }\end{array}$ & $\begin{array}{c}2020-21 \\
\text { PLAN }\end{array}$ & $\begin{array}{c}2021-22 \\
\text { PLAN }\end{array}$ & $\begin{array}{c}2022-23 \\
\text { PLAN }\end{array}$ \\
\hline \multicolumn{11}{|l|}{ UNDERGRADUATE } \\
\hline Distance (80-100) & $50 \%$ & $49 \%$ & $52 \%$ & $60 \%$ & $63 \%$ & $63 \%$ & $63 \%$ & $63 \%$ & $63 \%$ & $63 \%$ \\
\hline Hybrid (50-79) & $4 \%$ & $2 \%$ & $1 \%$ & $1 \%$ & $1 \%$ & $0 \%$ & $2 \%$ & $2 \%$ & $2 \%$ & $2 \%$ \\
\hline Classroom (0-50) & $46 \%$ & $49 \%$ & $47 \%$ & $39 \%$ & $36 \%$ & $37 \%$ & $35 \%$ & $35 \%$ & $35 \%$ & $35 \%$ \\
\hline
\end{tabular}

GRADUATE

\begin{tabular}{|c|c|c|c|c|c|c|c|c|c|c|}
\hline Distance (80-100) & $24 \%$ & $34 \%$ & $31 \%$ & $36 \%$ & $33 \%$ & $32 \%$ & $33 \%$ & $33 \%$ & $33 \%$ & $33 \%$ \\
\hline Hybrid (50-79) & $4 \%$ & $5 \%$ & $1 \%$ & $3 \%$ & $3 \%$ & $9 \%$ & $10 \%$ & $10 \%$ & $10 \%$ & $10 \%$ \\
\hline Classroom (0-50) & $72 \%$ & $61 \%$ & $68 \%$ & $61 \%$ & $64 \%$ & $59 \%$ & $57 \%$ & $57 \%$ & $57 \%$ & $57 \%$ \\
\hline
\end{tabular}

Note: Full-time Equivalent (FTE) student is a measure of instructional activity (regardless of fundability) that is based on the number of credit hours that degreeseeking students enroll. FTE is based on the standard national definition, which divides undergraduate credit hours by 30 and graduate credit hours by 24 . Distance Learning is a course in which at least 80 percent of the direct instruction of the course is delivered using some form of technology when the student and instructor are separated by time or space, or both (per 1009.24(17), F.S.). Classroom/Traditional, is a course in which less than 50 of the direct instruction of the course is delivered using some form of technology when the student and instructor are separated by time, space or both. This designation can include activities that do not occur in a classroom (ie, labs, internships, practica, clinicals, labs, etc) - see SUDS data element \#2052. *Percentages may not total 100 due to rounding. 